\title{
Visualization and Microanalysis of Susceptible Regions in Tubular Pharmaceutical Glass Vials Using Scanning Electron Microscopy
}

\author{
Youlong Ma, Charudharshini Srinivasan and Sau Lee \\ US Food and Drug Administration, Silver Spring, Maryland, United States
}

Studies to assess the risk of particulate matter for injectables have been done with several Type-I pharmaceutical glass vials [1]. To continue the investigation of quality issues in injectables, it is important to understand the condensation of alkali and its role in affecting the durability of vial. For pharmaceutical applications, tubular glass vial is desired because of its dimensional consistency, lower weight and good thermal conductivity [2]. Borosilicate Type-I tubular glass vials are generally used for primary packaging. However, the condensate produced in Type-I borosilicate glass vials may react with active pharmaceutical ingredient (API), disturb glass network and generate particulates [3]. In this case, the condensate on interior of glass vial could be used as a critical indicator of the pharmaceutical package susceptibility.

The condensation in pharmaceutical glass vials was investigated in conventional borosilicate Type-I glass vials from different sources and pure quartz glass vials as control. The vials were broken and gently cleaned by blowing air to remove the particles generated during breakage. The regions of interest were selected and examined with a field emission scanning electron microscope (FE-SEM, TESCAN Mira3) with energy dispersive spectroscopy (EDS, Oxford Max-80 SDD).

In this study, SEM was used as a powerful tool to visualize the condensate on four different types of glass vials. Compared to borosilicate glass vials, pure quartz vials have no additive, therefore the latter were not expected to generate condensate during vial conversion. Proper voltage $(5 \mathrm{kV})$ was used to visualize the condensates with both secondary electron (SE) and backscattered electron (BSE) detectors. The SE images show the topographical information of the condensate (Figure 1). The low contrast between uniform glass and condensate under SE images (Figure 1, top panel) indicate insignificant topographical features of these condensates. While the high contrast BSE images (Figure 1, bottom panel) demonstrate the distinct composition in condensate from glass, the dark condensate regions imply the enriched low atomic number elements and brighter regions indicate the accumulation of high atomic number elements. Figures $1 \mathrm{~d}$ and $1 \mathrm{~h}$ are confirmatory evidence for the homogeneity of the control pure quartz glass for the uniform elemental distribution as shown. EDS analysis (Figure 2) confirms the qualitative observation obtained from BSE images. The condensate from borosilicate glass vials show a steep drop in silicon (Si) and concentrated boron (B) and sodium (Na) at the location of the condensate (Figure 2. a-c), while the control pure quartz glass vial shows homogeneous surface under EDS analysis (Figure 2. d). This research provides an insight on the elemental distribution and susceptible regions in various pharmaceutical glass vials.

Disclaimer: This article reflects the views of the author and should not be construed to represent FDA's views or policies.
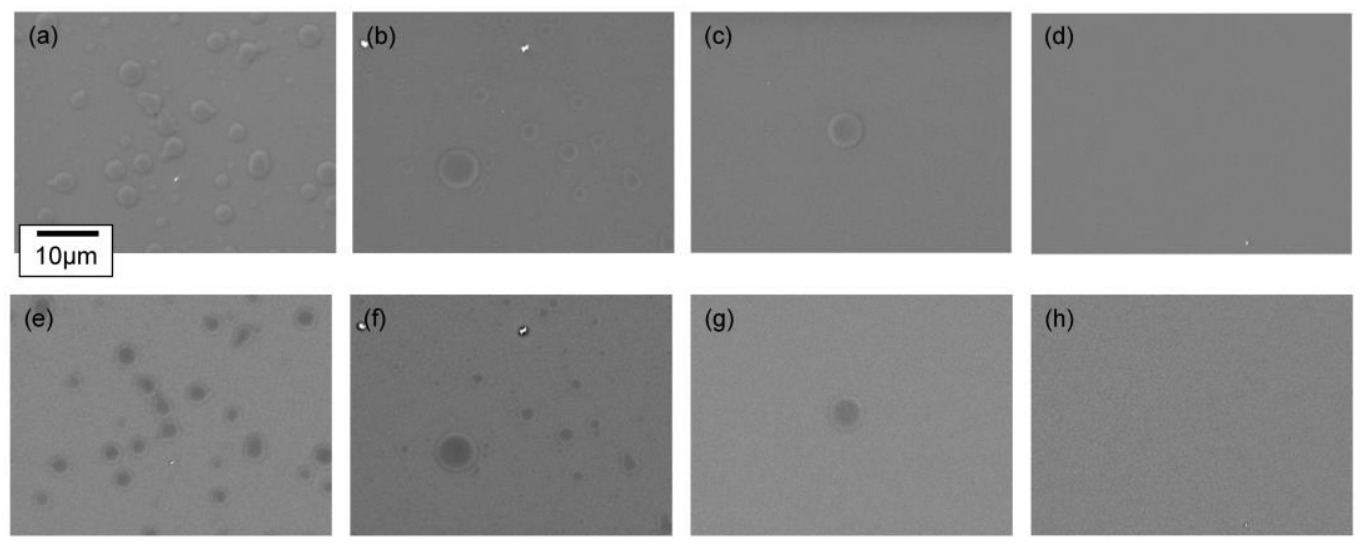
Figure 1. Images of secondary electron (SE) and backscattered electron (BSE) in glass vial interior: top panel SE images (a-d) and bottom panel BSE images (e-h) to demonstrate alkali rich and silicon rich regions.
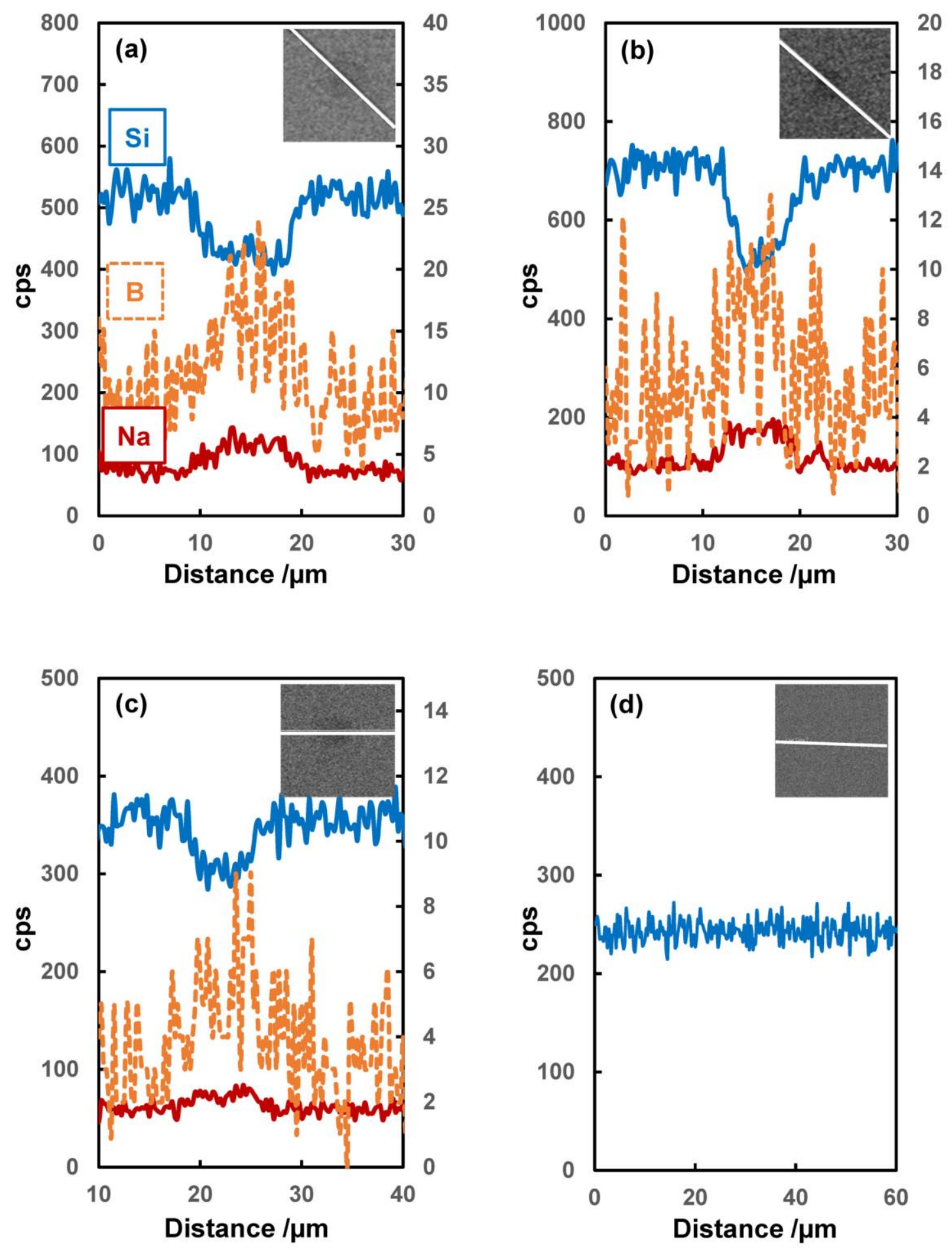

Figure 2. The EDS analysis of the condensates on the interior of vials. Figures (a-c) show boron and sodium rich region in the condensates in Type-I borosilicate glass vials, while (d) pure quartz glass vial 
show homogeneity on the inner surface structure. Solid lines in the figure for Si (blue) and $\mathrm{Na}$ (red) are shown on the primary axis; dashed line Boron (orange) is shown on the secondary axis. The subset images on each figure indicate the region scanned for EDS.

\section{References}

[1] C Srinivasan et al., (2019), p. 118510.

[2] RA Schaut and WP Weeks, PDA Journal of Pharmaceutical Science and Technology 71 (2017), p. 279.

[3] R Swift in "Pharmaceutical Dosage Forms - Parenteral Medications, Third Edition", ed. S Nema and JD Ludwig, (CRC Press, Boca Raton) p. 287. 